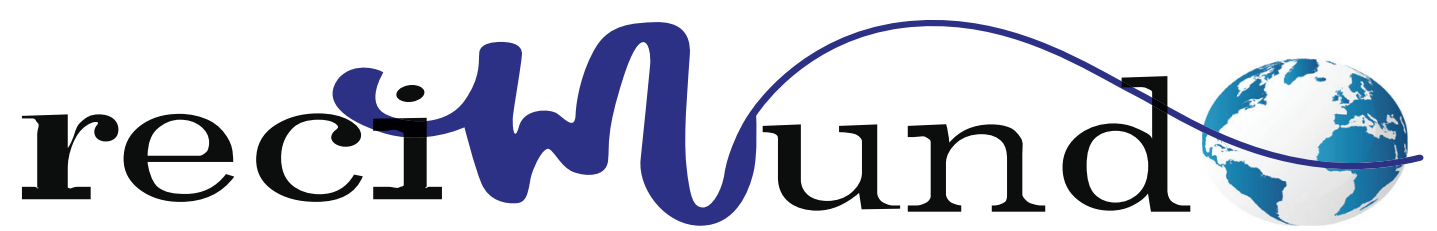

Revista Científica Mundo de la Investigación y el Conocimiento

DOI: 10.26820/recimundo/4.(1).enero.2020.236-348

URL: http://recimundo.com/index.php/es/article/view/756

EDITORIAL: Saberes del Conocimiento

REVISTA: RECIMUNDO

ISSN: 2588-073X

TIPO DE INVESTIGACIÓN: Artículo de Revisión

CÓDIGo UNESCO: 3201 Ciencias Clínicas; 3201.11 Radiología

PAGINAS: $236-248$

\title{
Situs inversus de vesicula biliar colecistectomía laparoscópica
}

\section{Situs inversus of gallbladder laparoscopic cholecystectomy}

\section{Situs inversus da colecistectomia laparoscópica da vesícula biliar}

Diana Carolina Mendoza Mendoza'; Lucio Hernando Sozoranga Morocho²; Juliana Katherine Mora Mendoza ${ }^{3}$

\section{RECIBIDO: 18/09/2019 ACEPTADO: 29/10/2019 PUBLICADO: 31/01/2020}

1. Médico; Investigador Independiente; Guayaquil, Ecuador; dianacarolinamendoza94@gmail.com; (DD https:// orcid.org/0000-0002-1449-7623

2. Magister en Gerencia de Salud para el Desarrollo Local; Doctor en Medicina y Cirugía; Investigador Independiente; Guayaquil, Ecuador; dr.Ihsm@hotmail.es; (D) https://orcid.org/0000-0001-6039-9170

3. Médico General; Investigador Independiente; Guayaquil; Ecuador, kathyta718@hotmail.com; (D) https://orcid. org/0000-0002-5030-450X

CORRESPONDENCIA

Diana Carolina Mendoza Mendoza

dianacarolinamendoza94@gmail.com

\section{Guayaquil, Ecuador}

(C) RECIMUNDO; Editorial Saberes del Conocimiento, 2020 


\section{RESUMEN}

Uno de los sistemas más importantes del cuerpo humano es el digestivo. Dentro de él se encuentra las vías biliares las cuales están conformadas principalmente por el hígado y la vesícula biliar. El hígado segrega bilis hacia las vías donde es transferida a la vesícula biliar. Cuando se produce la ingesta de alimentos, la vesícula biliar expulsa la bilis hacia el estómago para que pueda transformar los alimentos en las vitaminas y minerales necesarios para el buen funcionamiento del organismo. Ahora, si existe una alteración genética como el situsinversus, la cual se manifiesta en que los organismos, del aparato digestivo, por ejemplo, se localizan en la posición opuesta ejerciendo una situación tipo espejo, entonces produce modificaciones en el diagnóstico y tratamientos para las diferentes patologías referentes a las vías biliares y más si el tratamiento quirúrgico es con la colecistectomía laparoscópica. Por lo cual, esta investigación tiene como objetivo general analizar el fenómeno de situsinversus de vesícula biliar con colecistectomía laparoscópica. La metodología empleada se refiere a una investigación documental y bibliográfica. Los resultados se basaron en el análisis del situsinversus en el organismo, así como también el análisis de los diagnósticos y el tratamiento con colecistectomía laparoscópica para esta rara situación anatómica. Como conclusión, se obtuvo que el tratamiento de colecistectomía laparoscópica es la que brindamayor confianza, efectividad, es menos invasiva y produce menores daños colaterales, por lo que es la punta de lanza para cualquier tratamiento de las vías biliares; del mismo modo para aquellos pacientes consitusinversus, donde el protocolo de acción es cambiado en diferente orientación, y en la cual prevalece la experiencia del equipo médico en esta situación.

Palabras clave: Vesícula Biliar, Bilis, Situsinversus, Colecistectomía Laparoscópica.

\section{ABSTRACT}

One of the most important systems of the human body is the digestive. Inside it is the bile ducts which are mainly made up of the liver and gallbladder. The liver secretes bile into the pathways where it is transferred to the gallbladder. When food intake occurs, the gallbladder expels bile into the stomach so that it can transform food into the vitamins and minerals necessary for the proper functioning of the body. Now, if there is a genetic alteration such as situsinversus, which is manifested in that the organisms, of the digestive system, for example, are located in the opposite position exercising a mirror-like situation, then it produces modifications in the diagnosis and treatments for the different pathologies related to the bile ducts and more if the surgical treatment is with laparoscopic cholecystectomy. Therefore, this research has as a general objective to analyze the phenomenon of gallbladder situsinversus with laparoscopic cholecystectomy. The methodology used refers to a documentary and bibliographic investigation. The results were based on the analysis of situsinversus in the organism, as well as the analysis of diagnoses and treatment with laparoscopic cholecystectomy for this rare anatomical situation. In conclusion, it was obtained that the treatment of laparoscopic cholecystectomy is the one that provides greater confidence, effectiveness, is less invasive and produces less collateral damage, making it the spearhead for any treatment of the bile ducts; in the same way for those patients consitusinversus, where the protocol of action is changed in different orientation, and in which the experience of the medical team prevails in this situation.

Keywords: Gallbladder, Bile, Situsinversus, Laparoscopic Cholecystectomy.

\section{RESUMO}

Um dos sistemas mais importantes do corpo humano é o digestivo. No interior, estão os ductos biliares, compostos principalmente pelo fígado e pela vesícula biliar. O fígado secreta a bile nas vias onde é transferida para a vesícula biliar. Quando ocorre a ingestão de alimentos, a vesícula biliar expulsa bile no estômago, para que possa transformar os alimentos em vitaminas e minerais necessários ao bom funcionamento do corpo. Agora, se houver uma alteração genética, como situsinversus, que se manifesta no fato de que os organismos do sistema digestivo, por exemplo, estão localizados na posição oposta, exercendo uma situação semelhante ao espelho, produz modificações no diagnóstico e tratamentos pelas diferentes patologias relacionadas aos ductos biliares e mais se o tratamento cirúrgico for realizado com colecistectomia laparoscópica. Portanto, esta pesquisa tem como objetivo geral analisar o fenômeno de situsinversus da vesícula biliar com colecistectomia laparoscópica. A metodologia utilizada refere-se a uma investigação documental e bibliográfica. Os resultados foram baseados na análise de situsinversus no organismo, bem como na análise de diagnósticos e tratamento com colecistectomia laparoscópica para esta rara situação anatômica. Conclui-se que o tratamento da colecistectomia laparoscópica é aquele que proporciona maior confiança, eficácia, é menos invasivo e produz menos danos colaterais, tornando-a ponta de lança para qualquer tratamento dos ductos biliares; da mesma forma para aqueles pacientes consumados em inversão, onde o protocolo de ação é alterado em orientações diferentes e em que a experiência da equipe médica prevalece nessa situação.

Palavras-chave: Vesícula biliar, bílis, Situs Inversus, colecistectomia laparoscópica. 


\section{Introducción}

Existe dentro del organismo un proceso en la cual ayuda a realizar la digestión de los alimentos. Este producto es la bilis y se desplaza por toda la vía biliar. Es un líquido viscoso, de color verdoso-amarillento y sabor amargo, segregado por el hígado y vertido en el intestino por las vías biliares durante el período digestivo y almacenada en la vesícula(VISOR, 1997).

Además, es de reacción alcalina, la osmoralidades igual al de la sangre, se compone de agua en un 98\%, ácido cólico, desoxicólico y quenodesoxicólico (ácidos bilia- res), fotolipidos, colesterol, ácidos grasos, electrólitos, urea y ácido úrico, etc(VISOR, 1997). Es por ello, que juega un papel importante en el aparato digestivo, así como las vías biliares.

La via biliar esta conformada por dos vías la intrahepática y la extrahepática. La vía biliar intrahepática está formada por los canalículos segmentarios y la vía biliar extrahepática se origina habitualmente por la confluencia de los dos conductos hepáticos, derecho e izquierdo en la cara inferior del hígado para formar el conducto hepático común(MITIDIERI, 2020). En la Figura 1 se puede detallar la vía biliar.

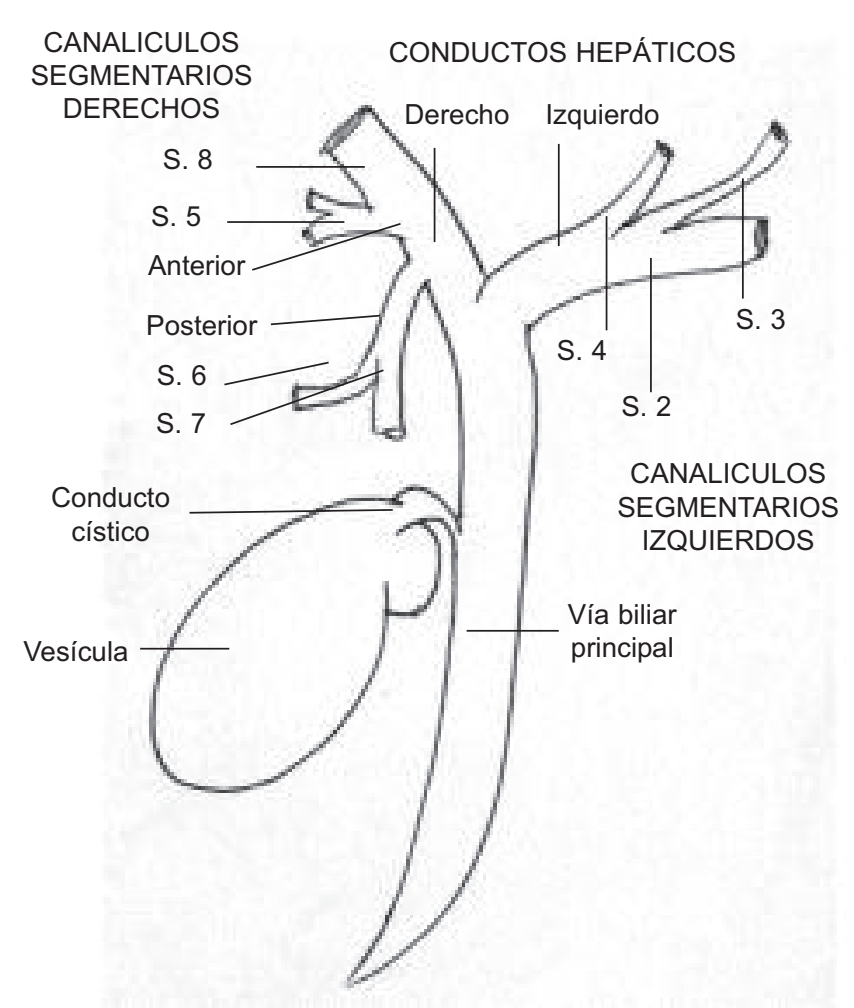

Figura 1. Vía biliar intra y extrahepática

Fuente: (MITIDIERI, 2020)

Dentro de esta vía biliar se encuentra la vesícula, la cual tiene la función de depositar la bilis segregada por el hígado. Su función particular consiste en que, al ingerir alimentos, el intestino segrega hormonas que produce una estimulación cuyo resultado es que la vesícula se contrae, vacía y suelta bilis en el intestino (duodeno) para disolver grasas y vitaminas liposolubles (A, D, E, K)
(Muñíz, 2020). En la Figura 2 se detalla la ubicación de la vesícula biliar. 


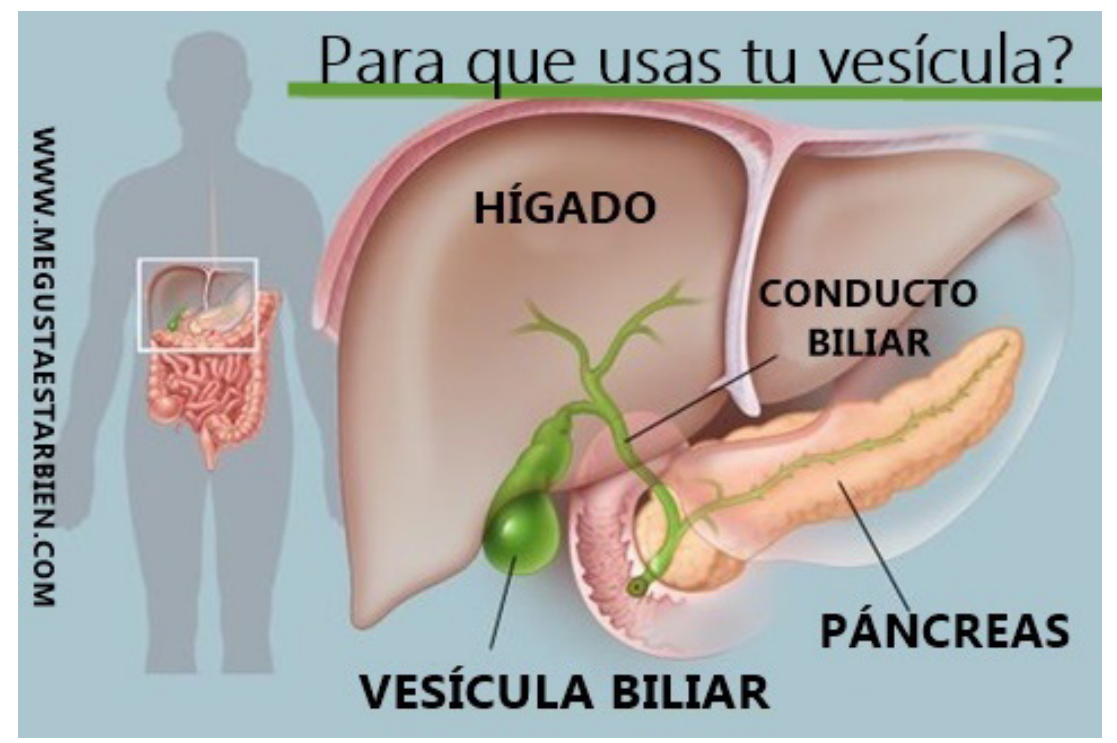

Figura 2. Ubicación de la vesícula biliar

Fuente: (Muñíz, 2020)

La vesículaestá conformada por 3 partes fundamentales, el fondo el cuerpo y el cue-
Ilo. En la Tabla 1 se describe cada una de estas partes.

Tabla 1. Permanencia Hospitalaria

\begin{tabular}{|c|c|}
\hline PARTES & CARACTERÍSTICAS \\
\hline Fondo vesicular & $\begin{array}{l}\text { Es la estructura sacular que excede el borde anterior hepático; se } \\
\text { proyecta en superficie a la altura del extremo anterior de la } 10^{a} \\
\text { costilla. }\end{array}$ \\
\hline Cuerpo & $\begin{array}{l}\text { Se relaciona con la cara inferior del hígado por su cara profunda, } \\
\text { poniéndose en contacto con la rodilla superior del duodeno por su } \\
\text { cara inferior. Está separado del hígado por una fascia vascular que } \\
\text { une entre sí las ramas de la arteria cística (fascia de Albanese). El } \\
\text { espacio entre la fascia de Albanese y la pared vesicular está } \\
\text { atravesado por las ramas arteriolares que se dirigen desde la arteria } \\
\text { hacia la pared vesicular; el espacio entre la fascia y la cara inferior del } \\
\text { hígado es en cambio avascular, y puede ser aprovechado para su } \\
\text { disección. Esto se puede visualizar en la Figura } 3 \text {. }\end{array}$ \\
\hline Cuello & $\begin{array}{l}\text { Es la porción que une al cuerpo con el conducto cístico. Presenta una } \\
\text { prominencia sacular, la bolsa de Hartmann, que se dirige hacia abajo } \\
\text { y atrás. }\end{array}$ \\
\hline
\end{tabular}

Fuente: (MITIDIERI, 2020) 


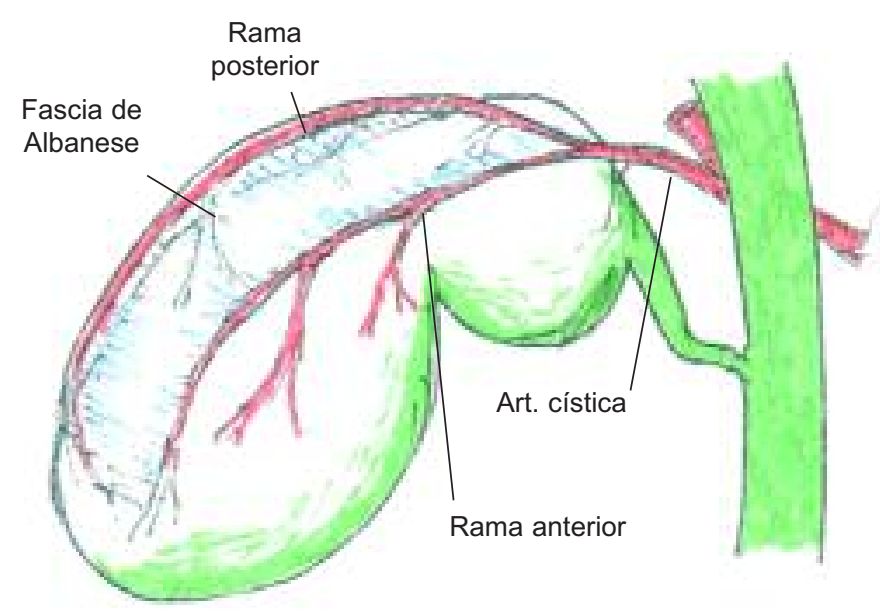

Figura 3. Partes de la vesícula biliar donde se observa la Fascia de Albanese

Fuente: (MITIDIERI, 2020)

Cuando en el organismo hay un desequilibrio en el mecanismo de funcionamiento ocurren las enfermedades. En vía biliar sucede que la presencia de cálculos produce diferentes trastornos. Cuando la secreción biliar se estanca en la vesícula y se produce la cristalización y precipitación de colesterol, sales biliares, lecitina o pigmentos aparecen los cálculos biliares donde en ocasiones se forma uno, pero con frecuencia su numero es considerable(Carbonell, Arteaga Prado, González, Ferro, \& Hernández, 2012).

A nivel mundial las enfermedades biliares tienen relación con la colelitiasis.Se define como la presencia de cálculos que se forman en las vías biliares que se encuentran en el hígado o en las vías de transporte de la bilis al tubo digestivo, los cuales son de tamaño, forma y composiciones variables(Ortiz \& Sánchez, 2018).

Ahora, cuando los cálculos se encuentran dentro de la vesícula ejerciendo presión a las vías biliares es la enfermedad llamada colecistitis. Es una inflamación de la pared de la vesícula biliar que se caracteriza por la presencia de fiebre y dolor que se localiza en el segmento superior derecho en el abdomen que puede llegar a esparcirse hacia el hombro derecho(Ortiz \& Sánchez, 2018). En la Figura 4 se puede observar la obstrucción realizada por el calculo lo cual determina la colecistitis. 


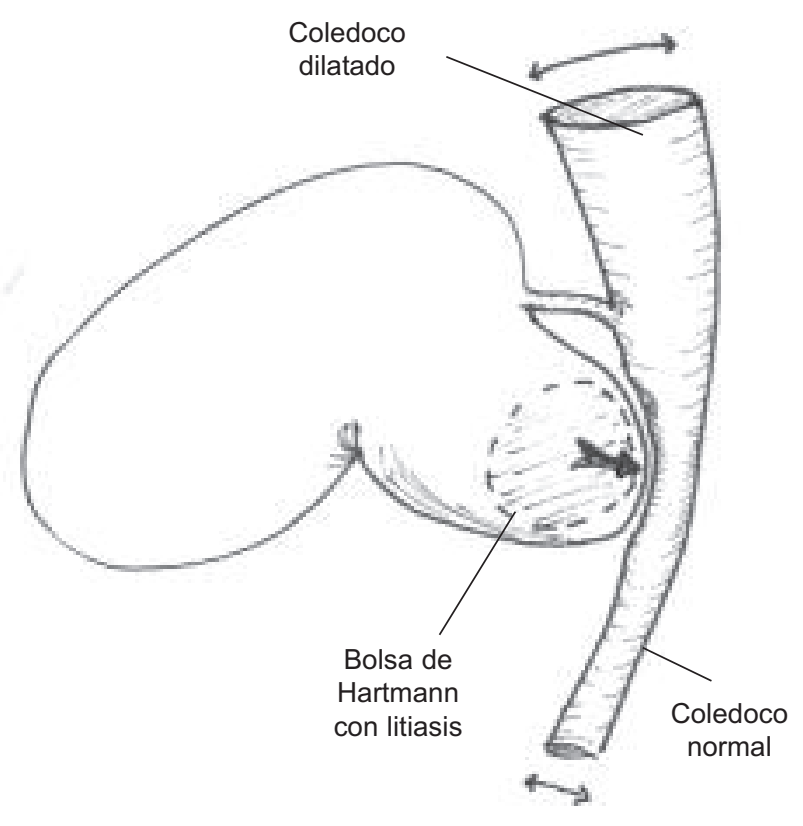

Figura 4. Compresión de la vía biliar por calculo vesicular

Fuente: (MITIDIERI, 2020)

Ahora los síntomas que se pueden presentar para estas patologías tienen algún parentesco. La colelitiasis se caracteriza por la presencia de hinchazón, irritación imprevista en la vesícula biliar que llega a causar dolor abdominal agudo, esta afección se produce cuando la bilis es retenida al interior de la vesícula biliar (Ortiz \& Sánchez, 2018).El dolor de la colecistitis persiste más tiempo y se puede acompañar de leucocitosis y fiebre(Motta Ramírez \& Rodríguez Treviño, 2010).

Existe dentro de esta patología biliar una rara enfermedad la cual es determinada solo cuando el paciente presente el dolor abdominal y acude a emergencias, por lo que el diagnóstico mediante $\mathrm{RX}$ o tomografía detalla la presencia de situsinversus.Es una anomalía congénita que puede ser total o parcial, con una prevalencia estimada de 1/10.000 nacimientos, caracterizado por la posición invertida de los órganos torácicos y abdominales con respecto al plano sagital(Ponce, Silva, \& Bolaños, 2018).Para el caso de la vesícula biliar, el situsinversus la somete a una posición no habitual en la parte abdominal por lo que su tratamiento no es común.

Por lo tanto, esta investigación tiene como objetivo general analizar el fenómeno de situsinversus de vesícula biliar con colecistectomía laparoscópica. La metodología empleada se refiere a una investigación documental y bibliográfica.

\section{Método}

Para realizar el desarrollo metodológico de empleo una serie de objetivos específicos basados en analizar la importancia de las vías biliares en el organismo, así como también comprender el situsinversus dentro del aparato digestivo, aunado a determinar el procedimiento para el diagnostico y tratamiento a las diferentes patologías que pueda sufrir la vesícula biliar cuando tiene esta situación anatómica inversa. Para ello, se desarrolló técnicas de investigación documentales y bibliográficas a través de textos, documentos y artículos científicos publicados disponibles en la web.

Resultados

\section{Definición de situsinversus}

Para poder describir esta enfermedad es

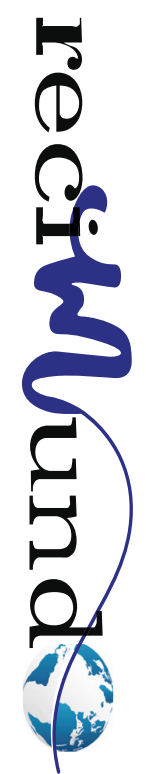


necesario poder determinar el significado de situs dentro de los organos del cuerpo humano. Este termino describe la posición de las auriculas cardiacas y las viceras abdominales con respecto a la línea media
(García \& Pinzón, 2018). En la Figura 5 se puede detallar la clasificación que se desprende del termino situs en el organismo.

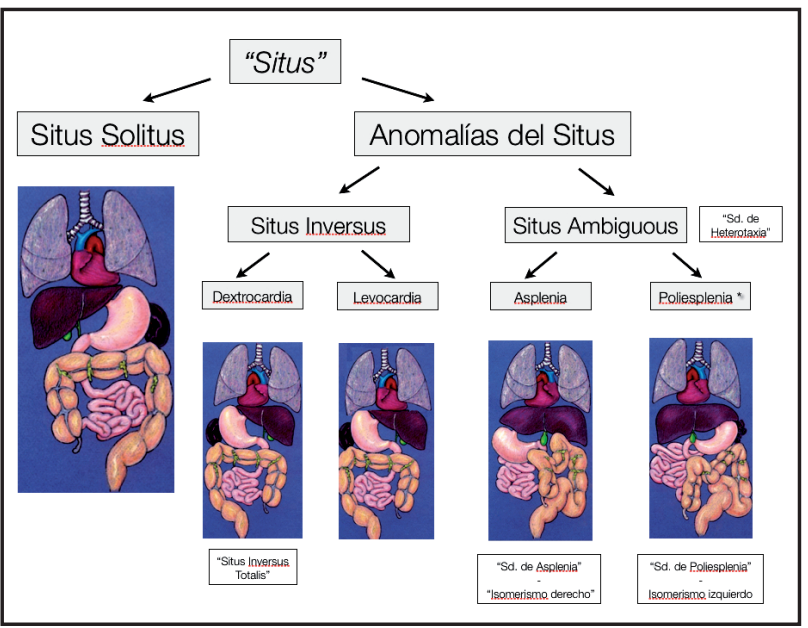

Figura 5. Clasificación en Situs Solitus y Anomalias del Situs

Fuente: (Corral G., A., \& Schiappacasse F., 2020)

Ahora, el situsinversus es una malformación congénita en un porcentaje mínimo de la población. Es de origen desconocido y de curso asintomático mayoritariamente, caracterizada por una modificación en la posición de uno o todos los órganos de cuerpo, lo que conforma una imagen en espejo(Alvarado Moncayo, 2018). El primer diagnóstico clínico de SitusInversus (SI), se efectuó en 1824, tras el desarrollo de la auscultación y percusión por Laennec y se atribuye a Vehsemeyer en 1897 el primer diagnóstico radiológico con rayos $\mathrm{X}$ (Ponce, Silva, \& Bolaños, 2018).

Es por ello, que para poder realizar un diagnostico eficiente para aquellos pacientes que tienen fuerte dolor abdominal es necesario, primordialmente, el origen de esta malformación. Estase debe a una alteración enel desarrollo embriológico durante la etapa de gastrulación(tercera semana), aunado a la alteración genética, autosómica recesiva, enel brazo largo del cromosoma 14, que afecta la cascada genéticaresponsable de la diferenciación izquierda-derecha(Carrillo Esper, y otros, 2012).La patología sue- le suceder mayormente en hombres que en las mujeres donde su presencia no influye en las expectativas de vida (Melchor-González, Pérez-García, Torres-Vista, \& Rodríguez-Brambila, 2000).

"EI ZIC3 es parte de la familia de genes ZIC. El gen codificada un factor de transcripción dedo de zinc fi y su papel en el desarrollo de la asimetría izquierda-derecha sigue siendo en gran parte desconocido, pese a que los modelos de ratones sugieren que ZIC3 puede interactuar con la vía de señalización nodal: también puede tener un rol en la regulación directa del desarrollo cardíaco y en el mantenimiento de la pluripotencialidad en células madre embrionarias. EI SIT se ha reportado en la mutación de ZIC3 en mujeres heterocigotas y se cree que la mayoría de los casos reportados en la literatura surgen de defectos en la función ciliar.

Existe una variación fenotípica marcada en los varones con heterotaxia ligada al $x$ asociada con mutaciones en $\mathrm{ZIC} 3$. Aunque no cabe descartar la presencia de la mutación en ZIC3 asociada al SIT, en la literatura, se 
presenta el primer caso de situsinversus asociado a una mutación ZIC3 en un varón. Esto confirma la penetrancia incompleta del gen". (Reales, Gallego, Espitia, Coley, \& Suárez, 2017, pág. 43)

El diagnóstico de situsinversus puede darse de manera incidental durante el transoperatorio o como hallazgo imagenológico(Alvarado Moncayo, 2018). En la Figura 6 se puede observar resonancia magnética potenciada en T2W, donde se detalla situsinversus en todo el aparto digestivo. Esta situacióndemanda especial atención en los médicos cirujanos, porque el diagnóstico en estos pacientes puede ser difícil, confuso e influir en la planificación de procedimientos invasivos y sobre todo en la de los procedimientos quirúrgicos(Melchor-González, Pérez-García, Torres-Vista, \& Rodríguez-Brambila, 2000).

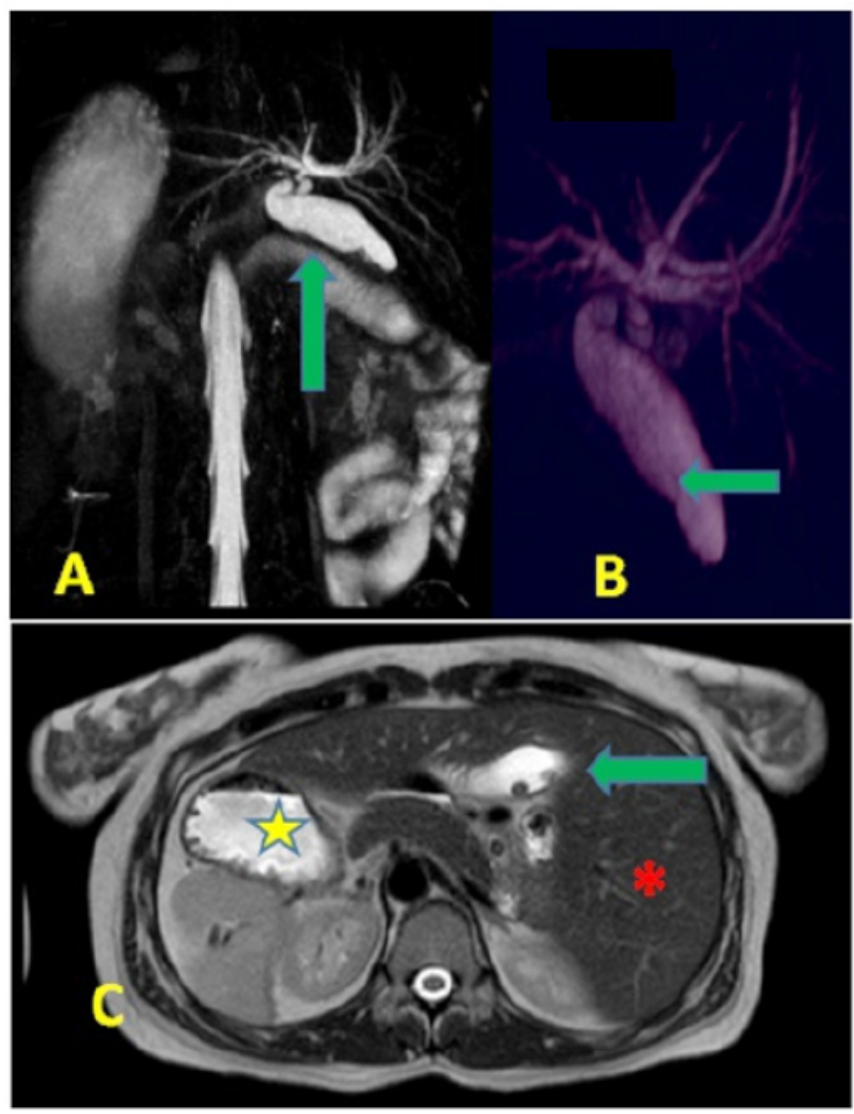

Figura 6. Resonancia magnética potenciada en T2W para diagnóstico de situsinversus Fuente: (García \& Pinzón, 2018)

Nota: Situs Inversus: Femenina de 27 años con diagnóstico de colelitiasisy situs inverso. Estudio solicitado para planeamiento quirúrgico. (A,B y C). Resonancia magnética potenciada en T2W en planos coronal(A) y axial (C) que demuestra imagen en espejo (C) del hígado $\left(^{*}\right)$.La vesícula biliar (A,ByC)seubica en el hipocondrio izquierdo(flechas verdes), también se observa imágenes hipointensas en suinterior (cálculos). El estómago se ubica en el hipocondrio derecho(estrella).Situs ambiguo (Isomerismo derecho). Femenina de 41 años condiagnóstico situs inverso, se desea evaluar vasos pulmonares. Seobserva el arco aórtico hacia el hemitórax derecho (flecha). Nótesetambién el hígado ubicado en el hipocondrio izquierdo (asterisco). 


\section{Vesícula biliaren pacientes con situsin- versus}

Cuando la vesícula tiene problemas se manifiesta a través de molestias y dolor. Ahora, si la vesícula se encuentra en un ambiente de situsinversusentonces los síntomas se muestran en el lado contrario a lo que realmente debe ocurrir. El dolor de la vesícula biliar puede encontrarse en el cuadrante superior izquierdo o en la línea media, estos fenómenos sugieren que el sistema nervioso no participa en esta transposición(Melchor-González, Pérez-García, Torres-Vista, \& Rodríguez-Brambila, 2000).
Seguidamente, se procede a evaluar el diagnostico a través de las diversas técnicas de imagen. El diagnóstico debe ser realizado antes del acto quirúrgico por sospecha en el examen clínico y corroborado por exámenes de gabinete (RX, USG y TAC)(Melchor-González, Pérez-García, Torres-Vista, \& Rodríguez-Brambila, 2000). Aunado, se deben realizar un electrocardiograma, una panendoscopía seriada esofagogastroduodenal, tránsito intestinal y coIon por enema(Valverde, y otros, 2010).En la Figura 7 se puede visualizar una TAC del abdomen en situsinversus.

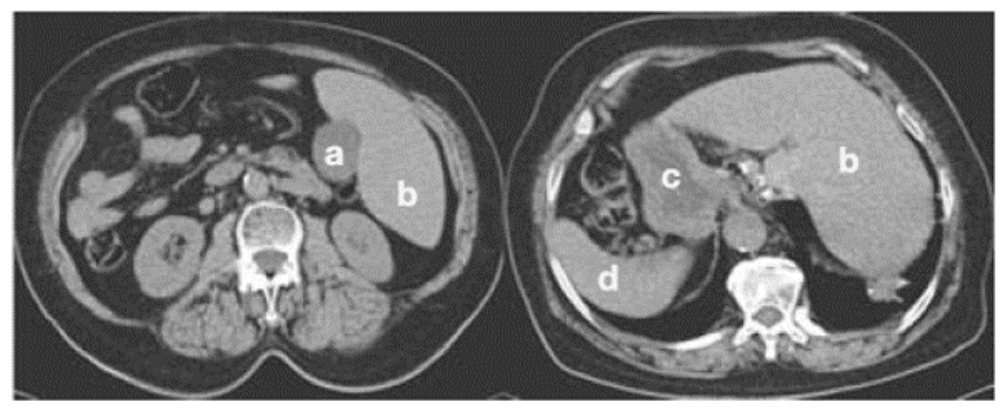

Figura 7. Tomografía computarizada de abdomen en situsinversustotalis. a) Vesícula biliar, b) hígado, c) estómago, d) bazo.

Fuente: (Pereira-Graterol \& Siso-Calderón, 2009)

Después de determinar la patología que pueda afrontar la vesícula biliar se recurre a unos d ellos tratamientos más seguros en la actualidad, la cirugía laparoscópica. En la actualidad se considera el procedimiento de primera eleccióndebido a que tiene grandes beneficios postoperatorios en función de diversos aspectos: menor inmunodepresión, mejor función pulmonar, menos dolor, menor estancia hospitalaria, rápida inserción a la actividad normal y ventajas estéticas(Morales, Moreno, \& Castillo, 2018).

"La colecistectomía laparoscópica es el tratamiento quirúrgico de elección en pacientes con situsinversus y colecistolitiasis sintomática; esto exige un conocimiento detallado de la anatomía y técnica quirúrgicas para ser capaz de practicar las maniobras en forma inversa. El cirujano puede efectuar el procedimiento modificando "en espejo" la técnica y no es necesaria la intervención adicional del ayudante sobre las conocidas misiones asignadas en un paciente con anatomía normal. La formal validación de las conclusiones, sin embargo, requiere el diseño de un estudio prospectivoque, dada la rareza de los casos, solo una serie multinstitucional puede proveer". (García-Núñez, Soto-Ortega, Payró-Hernández, \& R, 2008, pág. 151)

Lo primero que se debe realizar enel preoperatorio es la preparación del paciente antes de ingresar al quirófano, una explicación del proceso y los resultados, por parte del cirujano, permitirán una tranquilidad psicológica en el paciente. Posteriormente al ingresar al quirófano es necesario 
realizar las siguientes pautas: anestesia general, paciente en decúbito dorsal, posición americanay Trendelemburg invertido con lateralización a la derecha (Pereira-Graterol
\& Siso-Calderón, 2009). En la Figura 8 se detalla la posición americana en el quirófano para la realización de la colecistectomía laparoscópica.

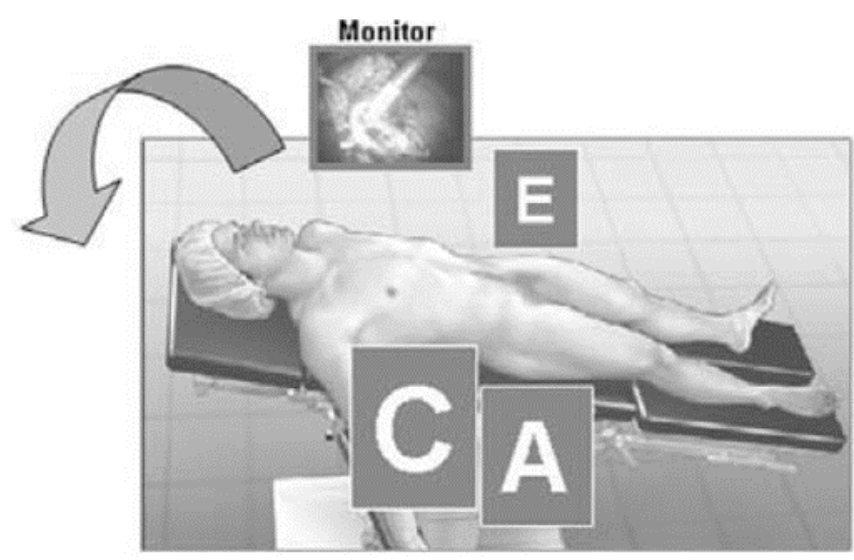

Figura 8. Esquema de la distribución del equipo quirúrgico: paciente en posición americana. C) Cirujano, A) ayudante, E), enfermera instrumentista.

Fuente: (Pereira-Graterol \& Siso-Calderón, 2009)

Luego, se procede a realizar el tratamiento quirúrgico tomando en consideración las partes del abdomen en modo situsinversus, tal cual se muestra en la Figura 9.

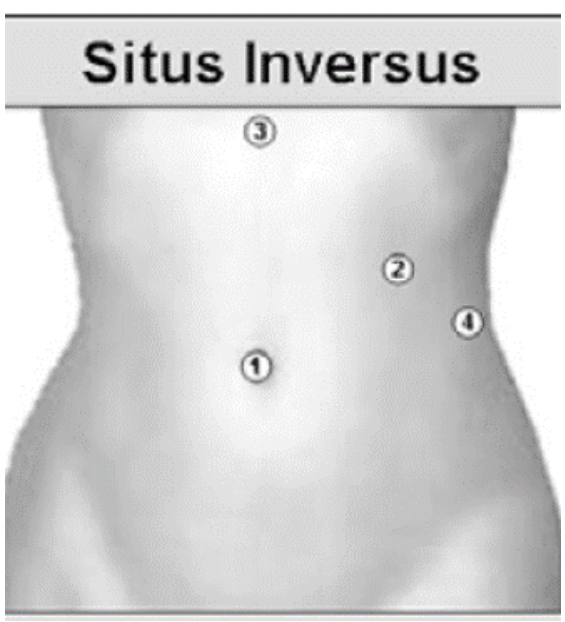

Figura 9. Distribución en la colocación de los puertos abdominales. 1. Lente. 2. Instrumentos de disección y corte (subcostal izquierdo con línea media clavicular). 3. Instrumento de tracción y movilización. 4. Instrumento para la tracción del fondo vesicular (flanco izquierdo con línea axilar anterior).

Fuente: (Pereira-Graterol \& Siso-Calderón, 2009, pág. 146)

"Abordaje umbilical con técnica abierta. Neumoperitoneo con CO2, hasta $12 \mathrm{~mm}$ Hg. Colocación de puerto umbilical de 10 $\mathrm{mm}$ e introducción del lente $\left(30^{\circ}\right)$ a través de este último. Colocación de los puertos restantes. Constatación de los hallazgos quirúrgicos, disección del triángulo de Calot, clipaje y sección del conducto y la arteria cística. Extracciónde la pieza quirúrgica a través de la incisión umbilical. Cierre del 
orificio del portal subxifoideo y correcciónde la hernia umbilical con vycril 1; piel con nailon 3-0. El tiempoquirúrgico fue de 60 minutos". (Pereira-Graterol \& Siso-Calderón, 2009, pág. 146)

Aunque la colecistectomía laparoscópica es una de las seguras y efectivas para tratar las diferentes patologías de la vesícula biliar es necesario tomar en cuenta ciertos factores de riesgo al momento de realizar el tratamiento quirúrgico. Estos son las lesiones vasculares e intestinales, pero también suelen presentarse lesiones vesiculares y uretrales, hernias e infecciones, entre otras de menor gravedad(Cárdenas, Laaz, Quinto, \& Rodríguez, 2019).

También, es necesario poder comprender los cuidados postoperatorios porque de ellos depende el éxito final del tratamiento. Estos cuidados son realizados en el hogar basados fundamentalmente en la predisposición del paciente y contando con el circulo familiar. El cuidado debe realizarse bajo una dieta baja en grasa, aumento del consumo de granos integrales, yogurt y quesos descremados,asimismo, impedir el consumo de granos cocidos, ayudarse con la alimentación de pescado, carne, pavo y pollo. Así como también evitar movimientos bruscos(Suscal, y otros, 2019).

\section{Discusión y conclusiones}

El aparato digestivo es uno de los sistemas más importantes del cuerpo humano. La vesícula biliar junto al hígado son parte fundamental en el proceso de digestión de la comida ingerida por el individuo. El hígado segrega la bilis la cual es almacenada en la vesícula biliar y cuando la persona ingiere los alimentos, la vesícula biliar expulsa la bilis hacia el estomago con el fin de transformar los alimentos en los nutrientes y las vitaminas necesarias para la energía que requiere el organismo. Es por ello, que el completo funcionamiento de la vesícula biliar es una parte fundamental para el desarrollo del ser.
Si la ingesta de alimentos no es balanceada, producto de alimentos con altos contenidos de grasa, la bilis no podrá cumplir efectivamente su trabajo por lo que producirá un desequilibrio manifestado en enfermedades donde predominan los cálculos. Estos estarán depositados dentro de la vesícula biliar o en las vías biliares produciendo manifestaciones de dolor en esa zona. Esta consecuencia hará realizar los protocolos médicos de diagnostico y tratamientos establecidos para cada patología.

Ahora, si existe desde el punto de vista alteraciones genéticas como el situsinversus, los procedimientos estandarizados pueden sufrir modificaciones. Esta situación produce que los órganosestén situados en sentido opuesto produciendo un efecto tipo espejo. Esto altera los procedimientos quirúrgicos estándar para los tratamientos de enfermedades biliares debido a que, principalmente, se requiere habilidad y destreza del especialista para que pueda existir buenos resultados. La manifestación del situsinversus es muy rara y ocurre en una pequeña población.

Los pasos a seguir para poder determinar la patología del paciente en presencia del situsinversus recae directamente en los diagnósticos por imágenes, desde RX, pasando por la TAC como protocolos para confirmar esta situación más los exámenes especializados en localizar el tipo de enfermedad como lo son una panendoscopía seriada esofagogastroduodenal, tránsito intestinal y colon por enema. De aquí, la junta médica diagnostica tratamiento quirúrgico como la colecistectomía.

En la actualidad la colecistectomía es realizada por laparoscopia en vez de a vierta porque brinda mayor confianza, efectividad, es menos invasiva y produce menores daños colaterales. La colecistectomíalaparoscópica es la punta de lanza para cualquier tratamiento de las vías biliares. El procedimiento para situsinversus es con el protocolo cambiado en diferente orientación. Para 
ello la experiencia del equipo medico prevalece en esta situación.

\section{Bibliografía}

Alvarado Moncayo, R. A. (2018). Case report: Situs inversus totalis. Revista Cubana de Cardiología y Cirugía Cardiovascular, 24(4).

Carbonell, C. L., Arteaga Prado, Y., González, T. P., Ferro, Y. P., \& Hernández, Z. H. (2012). Diagnóstico clínico y epidemiológico de la litiasis vesicular. Revisión bibliográfica. Revista de Ciencias Médicas de Pinar del Río, 16(1), 200-214.

Cárdenas, A. D., Laaz, S. A., Quinto, W. P., \& Rodríguez, J. D. (2019). Complicaciones de la cirugía laparoscópica. RECIAMUC, 3(2), 597-613.

Carrillo Esper, R., Delgadillo, C. R., Blanco, R. R., Graullera, M. G., Carrillo Córdova, C. A., \& Carrillo Córdova, D. M. (2012). Situs inversus totalis. Medicina Interna de México, 28(2), 187-191.

Corral G., G., A., L. W., \& Schiappacasse F., G. (11 de Febrero de 2020). Manifestaciones abdominales de las anomalías del Situs Ambiguous en el adulto. A propósito de cuatro casos. Obtenido de Revista Chilena de Radiología 2013; 19(1): 38-43.: https://scielo.conicyt.cl/pdf/rchradiol/v19n1/art07. pdf

García, E., \& Pinzón, A. (2018). Situs, Serie De Casos. Revisión Bibliográfica. Revista Médica de $\mathrm{Pa}$ namá-ISSN 2412-642X, 36(3)., 38-41.

García-Núñez, L., Soto-Ortega, L., Payró-Hernández, L., \& R, C.-P. (2008). Colecistectomía laparoscópica en situs inversus: la desventaja de ser diestro. Rev Gastroenterol Mex, Vol. 73, Núm. 3, 149-152.

Melchor-González, J. M., Pérez-García, R., Torres-Vista, M., \& Rodríguez-Brambila, V. R. (2000). Situs inversus. Report of two cases. Cirugía y Cirujanos, 68(2), 72-75.

MITIDIERI, V. C. (11 de Febrero de 2020). ANATOMÍA DE LA VÍA BILIAR. Obtenido de http://www. sacd.org.ar/ctreintaysiete.pdf

Morales, R. Z., Moreno, G. P., \& Castillo, M. C. (2018). Experiencia en colecistectomía laparoscópica en el Hospital Dr. Verdi Cevallos Balda Ecuador. QhaliKay. Revista de Ciencias de la Salud ISSN: 25880608, 2(2), 61-68.

Motta Ramírez, G. A., \& Rodríguez Treviño, C. (2010). Abordaje diagnóstico por imagen en patología benigna de la vesícula y vías biliares. Revista Mexicana de Cirugía Endoscópica, 11(2), 71-79.

Muñíz, A. (11 de Febrero de 2020). Vesícula, cál- culos biliares y dieta baja en grasa. Obtenido de http://megustaestarbien.com/2015/04/02/vesicula-calculos-biliares-y-dieta-baja-engrasa/

Ortiz, S. A., \& Sánchez, R. E. (2018). Influencia de los alimentos en las enfermedades de la vesícula biliar en el hospital básico latacunga del instituto ecuatoriano seguridad social. Revista Experiencia en Medicina del Hospital Regional Lambayeque, 4(3), 100-104.

Pereira-Graterol, F., \& Siso-Calderón, L. (2009). Consideraciones técnicas durante la colecistectomía laparoscópica en paciente con situs inversus totalis. Cirugía y Cirujanos, 77(2), 145-148.

Ponce, K. P., Silva, D. F., \& Bolaños, H. Y. (2018). SITUS INVERSUS TOTALIS: REPORTE DE UN CASO. Horizontes de Enfermería, (4), 7-14.

Reales, V. D., Gallego, G. C., Espitia, N. C., Coley, A. A., \& Suárez, O. G. (2017). Situs inversus totalis: revisión de tema con aproximación a la Genética y reporte de casos. Revista Colombiana de Cardiologia, 24(1), 40-47.

Suscal, N. R., Campoverde, J. L., Contreras, M. V., Carranza, L. H., Campuzano, P. G., \& Farfán, N. O. (2019). Colecistectomía laparoscópica cuidados postoperatorios. RECIAMUC, 3(3), 1284-1302.

Valverde, F. M., Ramos, M. J., Martínez, M. M., Montesinos, J. M., Rodríguez, M. E., Marín, M. R., \& Marín-Blázquez, A. A. (2010). Colecistectomía laparoscópica en una paciente con situs inversus totalis. Acta Gastroenterológica Latinoamericana, 40(3), 264-267.

VISOR. (1997). VISOR Enciclopedia Audiovisual. Tomo 3. Buenos Aires, Argentina: Plaza \& Janés Editores. 


\section{CITAR ESTE ARTICULO:}

Mendoza Mendoza, D., Sozoranga Morocho, L., \& Mora Mendoza, J. (2020). Situs inversus de vesicula biliar colecistectomía laparoscópica. RECIMUNDO, 4(1), 236-248. doi:10.26820/recimundo/4.(1).enero.2020.236-348

\section{(c) $(1) \otimes(\odot)$ BY NC SA \\ RECONOCIMIENTO-NOCOMERCIAL-COMPARTIRIGUAL CC BY-NC-SA ESTA LICENCIA PERMITE A OTROS ENTREMEZCLAR, AJUSTARY CONSTRUIR A PARTIR DE SU OBRA CON FINES NO COMERCIALES, SIEMPRE Y CUANDO LE RECONOZCAN LA AUTORÍA Y SUS NUEVAS CREACIONES ESTÉN BAJO UNA LICENCIA CON LOS MISMOS TÉRMINOS.}

